\title{
Retrospective epidemiological review on L. pneumophyla
}

\author{
Maria Antonietta Toscano', Angela Morello', Esmeralda Cammarata', Calogero \\ Rinzivillo², Mario Salmeri' \\ I Dipartimento di Scienze Bio-Mediche, Università degli Studi di Catania \\ 2 Dipartimento di Scienze Chirurgiche,Trapianti d'Organo e Terapie Avanzate, Azienda Ospedaliero-Universitaria, \\ Policlinico-Vittorio Emanuele, Catania
}

Key words: Legionella pneumophila, Nosocomial infections, Review

Studio epidemiologico retrospettivo su L. pneumophyla

\section{SUMMARY}

During the first annual control on hospital infections, was observed a curious but explainable trend regarding evidence of Legionella pneumophyla isolated by water systems in numerous public and private nosocomial structures.

After first massive isolation of $L$. pneumophyla strains at the beginning, a considerable reduction of isolates was noted, due to a program based on control and surveillance of water supplies, with regards to the aspectatives. But, in these last years a recurrent presence of isolates of $L$. pneumophyla was noted, despite all procedures used for the control of these bacteria.

From the first isolation, different epidemiological studies reported data about isolation of L. pneumophyla from water supplies in United States (1), Europe (5) and also in Italy (4), and relevant rule of this microorganism as etiologic agent of nosocomial infections is a well known sureness worldwide.

Also microbiological investigations performed during the hospital infection control program, started in 2000 by the Provincial Health Department of Catania showed a curious but understandable tendency on the presence of $L$. pneumophyla in water systems in many public and private nosocomial facilities.

In fact, with regards to the aspectatives, after the first massive isolation of L. pneumophyla in the early years, there has been a gradual significant reduction of its impact as a result of corrective actions taken and based on control and surveillance of water supplies $(2,3)$.

However, lately, despite all procedures used for the control of these bacteria there has been a striking resurgence in the frequency of isolation of $L$. pneumophyla, which has aroused considerable interest.

In order to highlight and then reverse this trend and to avoid future isolation of this organism does not comply with current legislation, we wanted to take back and compare all the previous data.

During this decade has been given particular attention to the presence of $L$. pneumophyla in water systems for 6 hospitals and 24 nursing homes.

Were first compared the results obtained in the years between 2001 and 2005. Similarly were collected and compared the results obtained from microbiological assays performed every year in the next five years, between 2006 and 2010 from the same facilities (Figure I).

Totally, during the two periods examined were analyzed 428 water samples from 30 facilities.

For the isolation was used the method described in ISO 11731 of 1998 (Water quality - Research and counting of L. pneumophyla) on Legionella BCYE medium and Legionella GVPC Selective agar.

For identification, the strains of L. pneumophyla were subjected to serotyping by Oxoid Legionella latex test kit (for serogroup 1, serogroups 2-14, and other species). Were not taken into account species of Legionella other than L. pneumophyla. A total of 60 strains were isolated from L. pneumophyla $(47 \%)$ in the first five years from 128 samples (Table 1) and 24 strains in the subsequent period from 300 samples, covering the years 
2006-2010 (8\%) (Table 2).

Of the 84 strains of L. pneumophyla isolated during ten years, $18(21.42 \%)$ belonged to serogroup 1 , the other $66(78.58 \%)$ to serogroups 2-14.

Statistical evaluation of two periods confirmed a very significanti "p" value ("p" $<<0,01 ; \chi^{2}$ test, Yates correction).

The striking reduction of the presence of $L$. pneumophyla in recent years could be explained by the attention shown by the health facilities and by the effectiveness of actions taken to reduce the unwelcome presence of this microrganism in the water.

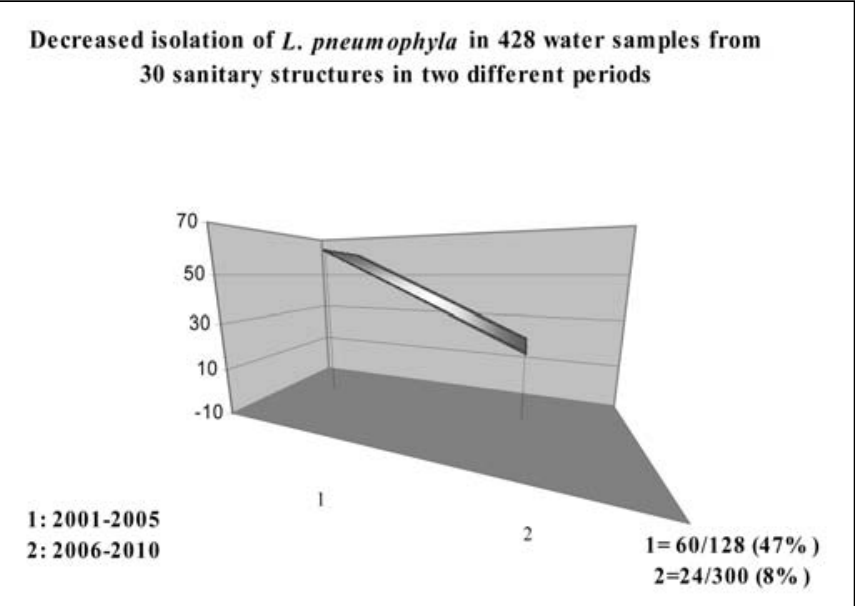

$P<0.01$

Figure $\mathbf{~}$.

Table I. Isolation of L. pneumophyla serogroup I (internal sectors) e serogroups 2-I4 (external sectors) indicated for years of first period (200I-2005)

Rates of isolation for each year: internal circle \% L. pneumophyla sg I (on 13 isolates),

external circle \% L. pneumophyla sg 2-14 (on 47 isolates)

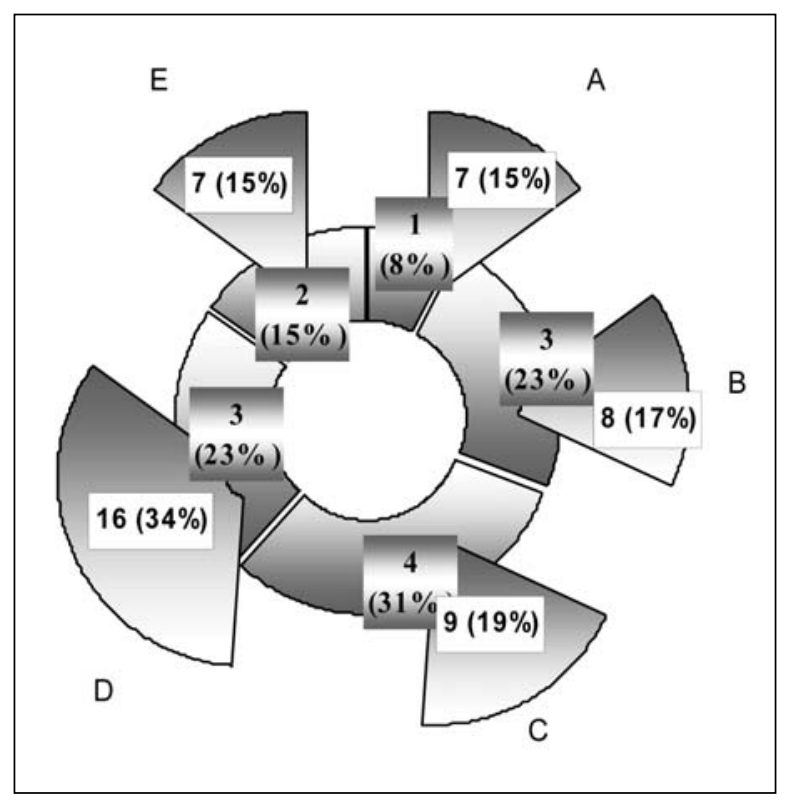

\begin{tabular}{rrr}
\hline sg. I & sg.2-14 & \\
\hline 1 & 7 & 2001 \\
\hline 3 & 8 & 2002 \\
\hline 4 & 9 & 2003 \\
\hline 3 & 16 & 2004 \\
\hline 2 & 7 & 2005 \\
\hline 13 & 47 & \\
\hline
\end{tabular}

Table 2. Isolation of L. pneumophyla serogroup I (internal sectors) e serogroups 2-14 (external sectors) indicated for years of second period (2006-2010)

\section{Rates of isolation for each year:} internal circle \% L. pneumophyla sg I (on 5 isolates), xternal circle \% L. pneumophyla sg 2-14 (on 19 isolates)

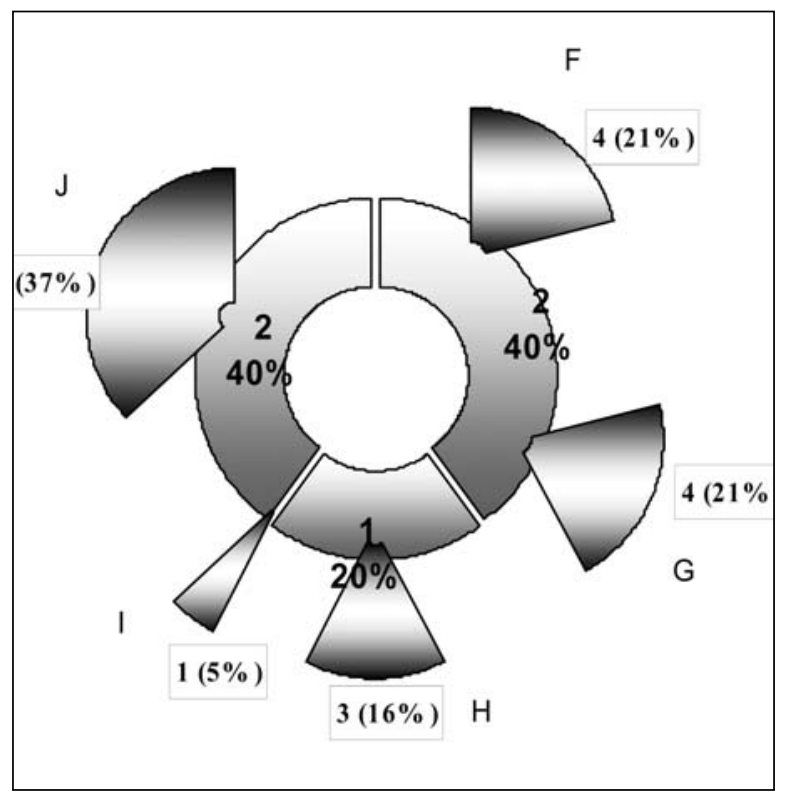

\begin{tabular}{rrr}
\hline sg.I & sg.2-I4 & \\
\hline 2 & 4 & 2006 \\
\hline$I$ & 4 & 2007 \\
\hline 0 & 3 & 2008 \\
\hline 0 & 1 & 2009 \\
\hline 2 & 7 & 2010 \\
\hline 5 & 19 & \\
\hline
\end{tabular}

$F=2006, G=2007, H=2008, I=2009, J=2010$ 


\section{REFERENCES}

1. Fields BS, Benson RF, Besser RE. Legionella and Legionnaire's disease: 25 years of investigation. Clin Microbiol Rev 2002 Jul; 15 (3): 506-26.

2. Guidelines for laboratories with activity of diagnostic microbiology and control of Legionella. Permanent Conference "Rapporto Stato e Regioni", (art 4, Decr. Legs. 28/8/1997, n. 281), between Ministery of Wellfare and Governors of Regions and Autonome Provinces. Gazzetta Ufficiale della Repubblica Italiana, 5/2/2005, n.28.

3. Guidelines for Prevention and Control of Hospital ISS and Ministery of Wellfare Permanent Conference for cooperation between "Stato, Regioni e Province autonome" of Trento and Bolzano dated 4/4/2000 Gazzetta Ufficiale della Repubblica Italiana, 5.5.2000, n. 103 Serie Generale.

4. Napoli C, Fasano F, Iatta R, Barbuti G, Cuna T, Montagna MT. Legionella spp. and legionellosis in southeastern Italy: disease epidemiology and environmental surveillance in community and health care facilities. BMC Public Health 2010; Nov 2; 10: 660 .

5. O’Neill E, Humphreys H. Surveillance of hospital water and primary prevention of nosocomial legionellosis: what is the evidence? J. Hosp. Infect. 2005, Apr; 59 (4): 273-9. 